\title{
The Beam Dump eXperiment
}

\author{
Luca Marsicano*, for the BDX Collaboration. \\ Università di Genova, INFN Genova \\ E-mail: luca.marsicanodge.infn.it
}

The Beam Dump eXperiment (BDX) is an electron-beam thick-target experiment aimed to investigate the existence of Light Dark Matter (LDM) particles in the MeV-GeV mass range. The experiment is expected to run at Jefferson Lab in a dedicated underground facility located $20 \mathrm{~m}$ downstream of the Hall A beam-dump, receiving up to $10^{22} 11 \mathrm{GeV}$ electrons on target in $\sim 1$ year time. The detector consists of a $\mathrm{CsI}(\mathrm{Tl})$ electromagnetic calorimeter (Ecal) and a veto system used to suppress the background. The expected signature of the DM interaction in the Ecal is a $\sim \mathrm{GeV}$ electromagnetic shower paired with a null activity in the surrounding active veto counters. A complete small-scale prototype of the final detector has been built in order to validate the proposed technology and evaluate the cosmogenic background rejection capability. Beam-related background has been estimated by means of Monte Carlo (MC) simulations. This paper describes the BDX experimental setup and the results of the background studies along with the status and perspectives of the experiment.

XXXIX International Conference on High Energy Physics

July 4-11, 2018

COEX, Seoul

${ }^{*}$ Speaker. 


\section{Introduction}

The search of Dark Matter (DM) is one of the hottest topics of modern physics. Despite the copious number of astrophysical and cosmological observations proving its existence, its elementary properties remain to date unknown [1]. Experimental efforts have been mainly focused, until today, in the WIMPs search (Weakly Interacting Massive Particles): particles with mass in the $100-1000 \mathrm{GeV}$ range, expected to weakly interact with SM particles [2]. However, no unequivocal evidence of WIMPs has been found to date. Recently, the interest in scenarios predicting the existence of light hidden sector particles (mass in the $\mathrm{MeV}-\mathrm{GeV}$ range) is growing. The simplest hidden sector model introduces one extra U(1) gauge symmetry [3] and a new gauge boson, the Dark Photon $\left(A^{\prime}\right)$. The interaction between the hidden sector and the SM is generated effectively by a kinetic mixing mechanism between the SM photon and the $A^{\prime}$ :

$$
\mathscr{L}=-\frac{1}{4} F_{\mu v}^{\prime} F^{\prime \mu v}+\frac{\varepsilon}{2} F_{\mu v} F^{\prime \mu v}+\frac{m_{A^{\prime}}^{2}}{2} A_{\mu}^{\prime} A^{\prime \mu}+g_{D} A_{\mu}^{\prime} J_{D}^{\mu}
$$

Here, $F_{\mu \nu}^{\prime}$ is the $A^{\prime}$ field strength, $g_{D}$ is the dark gauge coupling, $J_{D}^{\mu}$ is the current of DM fields and $\varepsilon$ parametrizes the degree of kinetic mixing between dark and visible photons. This scenario offers well-motivated opportunities for experimental exploration.

\section{Experiment Overview}

BDX will make use of the CEBAF (Continuous Electron Beam Accelerator Facility) high intensity electron beam, impinging on the Jefferson Lab Hall-A beam dump, placed at the end of the beam transport line. CEBAF can provide $11 \mathrm{GeV}$ electrons with a maximum current of about
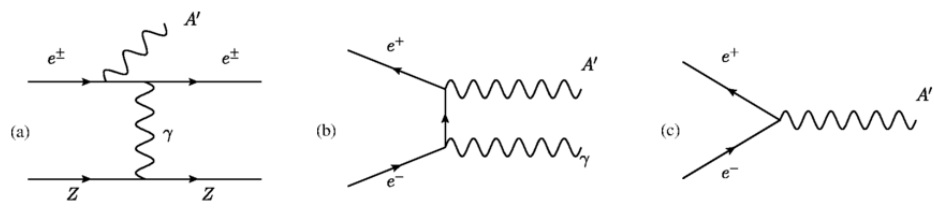

Figure 1: Processes producing $A^{\prime}$ s in the beam dump.

$65 \mu \mathrm{A}$, allowing BDX to collect $10^{22}$ electrons on target (EOT) in 285 days. As shown in Fig. 1 , the interaction of the beam in the dump can produce a Dark Photon $\left(A^{\prime}\right)$ through a bremsstrahlunglike process or through the annihilation of secondary positrons [4]. The $A^{\prime}$ s subsequently decay into pairs of DM particles $\bar{\chi} \chi$, which travel unaltered towards the BDX detector, placed $20 \mathrm{~m}$ downstream the beam dump. The detector is composed of an electromagnetic calorimeter (Ecal) of $\sim 800 \mathrm{CsI}(\mathrm{Tl})$ crystals unclosed into a $5 \mathrm{~cm}$ thick lead shielding and two active veto layers made of plastic scintillator to reject cosmic background; both crystals and vetos are read by SiPMs. Here, $\chi$ particles can scatter off electrons in the crystals giving rise to an electromagnetic shower. A passive shielding made of iron and concrete blocks is placed between the dump and the detector, in order to stop all SM particles (except neutrinos) produced in the dump that could reach the detector and interact mimicking the signal. The number of expected background events assuming an energy threshold of $350 \mathrm{MeV}$ is $\sim 5$ for the whole run. In case of no measured excess over the 
expected background, the accumulated data would provide stringent limits on the DM parameter space. Figure 2 shows the expected BDX reach in the $y-m_{\chi}$ parameter space, where $y$ is the dimensionless combination [5]:

$$
y=\varepsilon^{2} \alpha_{D}\left(\frac{m_{\chi}}{m_{A^{\prime}}}\right)^{4}
$$

The background evaluation as well as the optimization procedure of the shielding and the detector are described in the following sections.

\section{Background Evaluation}

\subsection{Cosmic Background}

Cosmogenic background consists mainly of muons and neutrons produced in the upper atmosphere. These particles can hit the detector, producing a signal in the Ecal. This background was measured with a prototype detector composed of a single $\mathrm{CsI}(\mathrm{Tl})$ crystal enclosed into two plastic scintillator layers, used as veto. Cosmogenic background was measured at Laboratori Nazionali del Sud (Catania), in a condition similar to that one expected for the full scale experiment at JLab. The results of this measurement were used to extrapolate the expected number of cosmic background events for BDX. For a detailed review of the BDX prototype measurment campaign, see [5] .

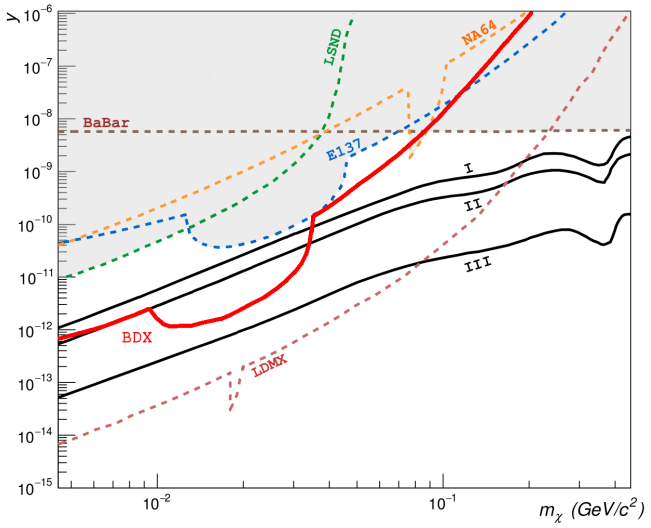

Figure 2: Red line shows the $90 \%$ CL exclusion limit for BDX. Light gray indicates limits set by previous experiments.

\subsection{Beam Related Background}

Beam related background was estimated using FLUKA and GEANT4 simulations. The geometry of the BDX underground facility was implemented in the simulation framework, including the iron and concrete shielding between the dump and the hall. The flux of SM partcles (muons, neutrons, photons and neutrinos) reaching the BDX detector was evaluated through massive FLUKA simulations $\left(10^{17}\right.$ equivalent EOT), for various configurations of the shielding. This study allowed us to define the best shielding setup, for which all SM particles except neutrinos are stopped before reaching the underground hall [6]. Regardless of the shielding, a sizeable number of neutrinos $\left(v_{e}, \bar{v}_{e}, v_{\mu}\right.$ and $\left.\bar{v}_{\mu}\right)$ reaches the BDX detector. Among these, a small but non negligible fraction has energy higher than $500 \mathrm{MeV}$. These events may produce a signal in the BDX detector similar to a DM interaction. To estimate this background we followed a multi-step procedure: FLUKA was used to generate neutrinos in $11 \mathrm{GeV}$ electron/dump interaction, propagate them to the BDX location and sample particles produced in the interaction with the detector $\mathrm{CsI}(\mathrm{Tl})$ crystals. Reaction products were then fed to GEANT4 code that contains a detailed and realistic description of the BDX detector response. 


\section{Detector Optimization}

To estimate the BDX reach, the detector response to signal events was evaluated through GEANT4 simulations. A custom generator was used to generate events within the detector volume, considering the interaction process with atomic electrons. To optimize the detector design,
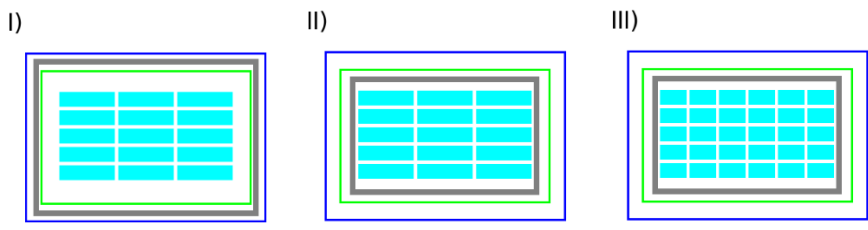

Figure 3: Schematic of the three detector configurations tested (lateral view): crystals are drawn in cyan, internal veto in green, external veto in blue and lead in gray. The position of the lead shielding with respect to the vetos was modified (I and II), as well as the dimension of the crystals (III). In the best configuration identified (II), the lead shielding is placed within both veto layers.

we tested three different configurations, slightly varying the components arrangement (see Fig. 3). For each detector variation, signal and neutrino background were simulated, and events were reconstructed and analyzed applying different set of selection cuts on the reconstructed variables [6]. This study allowed us to identify the detector configuration and cuts set resulting in the best reach for the experiment [6].

\section{Conclusions}

BDX is an electron beam thick-target experiment at JLab aiming to search for LDM particles in the $\mathrm{MeV}-\mathrm{GeV}$ mass range. The sensitivity of BDX was evaluated by measuring the cosmic background with a prototype under conditions similar to those proposed at JLab and estimating the beam-related background using FLUKA and GEANT4 MC simulations. In case of no measured excess over the expected background, BDX will be able to provide new stringent limits on the DM parameter space.

\section{References}

[1] K. A. Olive et al., Review of Particle Physics. Chin. Phys., C38:090001, (2014).

[2] Annika H. G. Peter, Dark Matter: A Brief Review. (2012), arXiv:1201.3942 [astro-ph.CO].

[3] B. Holdom, Two U(1)'s and $\varepsilon$ charge shifts, Phys. Lett. B, 166B, 196 (1986).

[4] L. Marsicano et al., Novel Way to Search for Light Dark Matter in Lepton Beam-Dump Experiments, Phys. Rev. Lett. 121, 041802, (2018), arXiv:1807.05884 [hep-ex].

[5] M. Battaglieri et al., Dark matter search in a Beam-Dump eXperiment (BDX) at Jefferson Lab, (2016), arxiv:1607.01390v1.

[6] M. Battaglieri et al., Dark matter search in a Beam-Dump eXperiment (BDX) at Jefferson Lab - 2018 update to PR12-16-001, (2018) 